\title{
Mechanisms and emergent properties of social structure: the duality of actors and social circles
}

\author{
Demival Vasques Filho \\ Digital Humanitites Lab, Leibniz-Institut für Europäische Geschichte \\ 55116 Mainz, Germany \\ E-mail: vasquesfilho@ieg-mainz.de.
}

I propose a theory of social structure that challenges the widely accepted role of preferential attachment and triadic closure as primary mechanisms of network formation. For this, I build upon Feld's concept of social circles, Breiger's concept of the duality of actors and groups, and Hinde's concept of interactions and relationships. The theory emphasizes that ties between actors arise and evolve according to social circles and social situations in which they participate, a notion straightforwardly modeled through two-mode and projected networks. Using recent results aided by analyses of empirical and artificial networks, I argue that structural properties such as tie strength, heterogeneity of popularity and strength among actors, clustering, community formation, and segregation emerge from homophily, jointly with overlap and social activity—mechanisms introduced in this study. The mechanisms form the two-mode network, and these structural properties naturally arise in the one-mode projection. The results show that social circle and social situation size distributions modulate network structures by interweaving with social 
activity distributions, and that overlap increases segregation from a network viewpoint. This theory's implications are broad, affecting several social processes ranging from social cohesion, tolerance, and child development to the spread of infectious diseases.

\section{Introduction}

The spread of knowledge, infectious diseases, innovation, misinformation, and other processes depends on patterns of social relations. It is a chain: the more we perceive these patterns, the better we understand the social processes, resulting in improved policies. For instance, the fact that governments worldwide are struggling to find a balance between socioeconomic disruption and halting the spread of COVID-19 shows that we still know too little about social structure to take adequate measures. The literature lacks theoretical approaches to the formation of large-scale networks, restricting itself to small-scale frameworks that depend on the feasibility of rich data collection or the implementation of statistical models. A more realistic approach to general population structures - one that provides better insights into social relations in different societies - is crucial to enhance data collection methods, study design in several fields, and policy-making.

This study attempts to address general population structures by proposing a theory focused on a long-standing, central, open question in social network research: how macrolevel structural properties emerge from micro-level mechanisms of network formation. A large body of research has made ubiquitous the mechanisms known as preferential attachment, triadic closure, and homophily, and has associated them respectively with degree heterogeneity, high clustering, and segregation (see the comprehensive review by Rivera, Soderstrom, and Uzzi (1) as well as other recent publications (2-10). However, a consensus on how these mechanisms function and interweave seems distant. For example, 
while Block (8) argues that homophily and triadic closure have "negative interaction," Asikainen et al. (9) claim that they present "cumulative effects," while Wimmer and Lewis (11) suggest that homophily can be "amplified" by triadic closure and other balancing mechanisms.

I argue that disagreements such as the above originate in the emphasis that social network research has placed on dyadic relations, an incomplete approach that fails to provide more insights into social structure at a large scale. Even though humans live mostly in groups - not only in pairs - the recent scholarship often overlooks relationships (or interactions) between individuals in the context of their social circles (or social situations), in contrast to previous suggestions (12-14). In their seminal review, McPherson, Smith-Lovin, and Cook (15) stated that "school, work, and voluntary organizational foci provide the great majority of ties that are not kin (Louch 2000:53), supporting Feld's (1981, 1982,1984) argument that focused activity puts people into contact with one another to foster the formation of personal relationships." Grossetti (16) reached the same conclusion in his empirical study in the Toulouse area: "The majority of relations thus arise from circles." Nonetheless, the role of social circles, and the duality of actors and groups in the formation of social structure, is still undervalued.

Based on Feld's (14) focus theory, Breiger's (17) concept of duality, and Hinde's (18) perception of interactions and relationships, I propose a theory of social structure that spotlights social circles and situations, thus challenging preferential attachment and triadic closure as the primary mechanisms of network formation. The theory reinforces the protagonism of homophily and introduces novel mechanisms, namely, social activity and overlap. The core concept of this approach is that relationships between actors stem mostly from their belonging to or participation 1 in social circles and social situations.

\footnotetext{
${ }^{1}$ For simplicity, I use the single term participation to refer to belonging, membership, affiliation, and
} 
In his work, Feld (14) called social circles an "extra-network social structure." Instead, the present theory considers social circles and situations to be another type of entity in the network. To model social structure, I use two networks: a two-mode network, connecting actors to circles and situations; and its one-mode projection, connecting pairs of actors if they participate together in at least one circle (17). The micro-level mechanisms act to form the two-mode network; through projection, the macro-level structural properties we observe in social networks naturally arise in the one-mode network (Fig. 1).

\section{Social structure, circles, and situations}

I use Robert Hinde's (18) concepts of interaction and relationship to define social situation and social circle. According to Hinde (18), an interaction "involves two or more individuals and one or more types of behaviour. It can be characterised in such terms as 'A does $\mathrm{X}$ to B' or 'A does X to B and B responds with Y'.' Here, I consider a social situation to be a short-term event where a sequence of positive, neutral, or negative interactions takes place, such as a date, a family dinner, a political debate, or closing a business deal with a stranger. The existence and quality of a social situation directly relates to psychological and physiological variables, following Hinde's (18) proposition regarding how these affect interactions.

Concerning relationships, I depart a little from Hinde's idea that "relationships involve a succession of interactions between two individuals" (18). In my view, the quality of a relationship indeed depends on a succession of social situations and relationships emerge from individuals participating in social circles (Feld 1981; McPherson, Smith-Lovin, and Cook 2001), but these relationships are created at the moment an individual joins the circle. The social circle is a long-term entity that provides the environment for a succession others. 


\section{Social structure model}

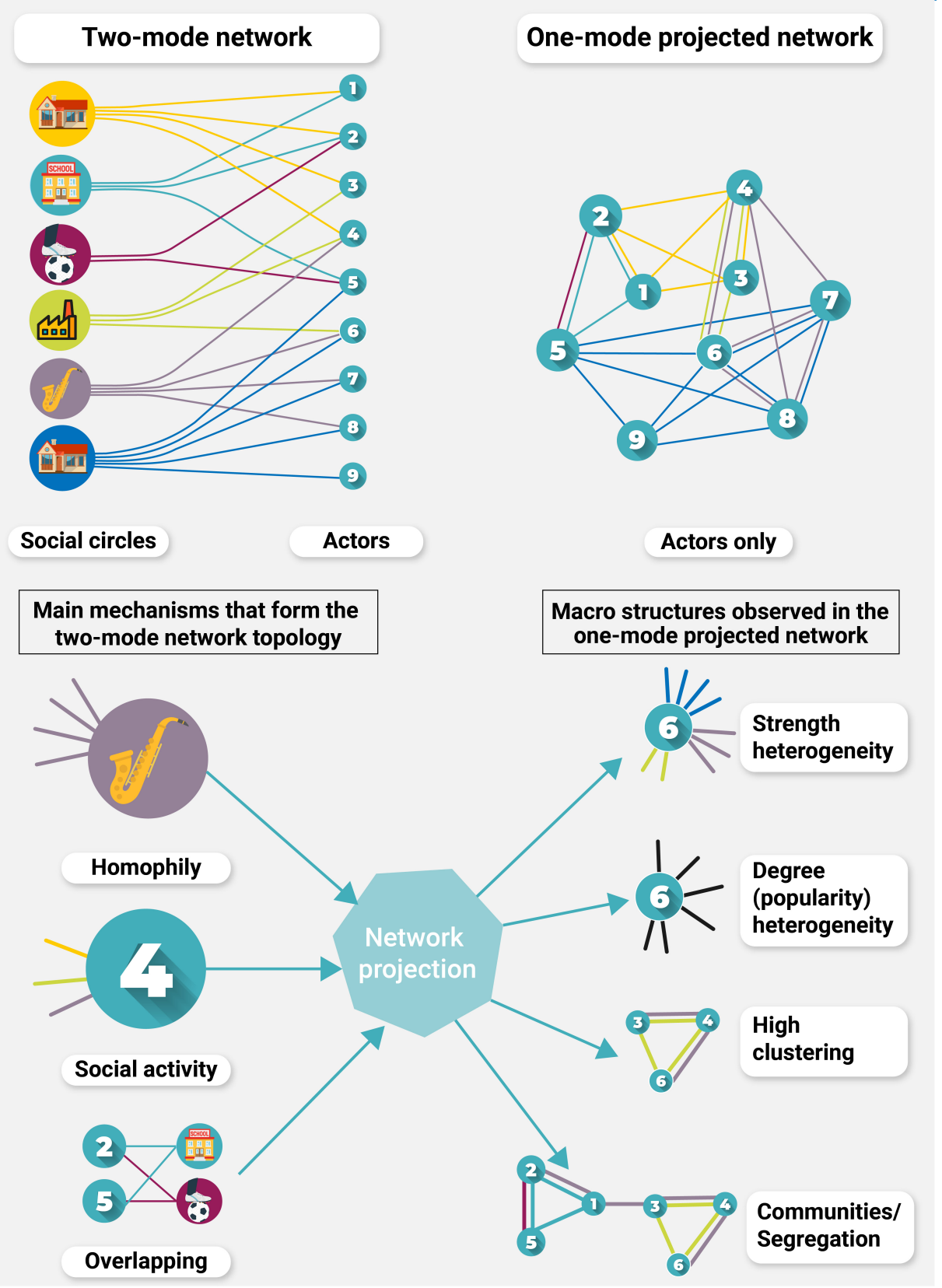

Figure 1: Diagram of social structure. Homophily, social activity, and overlap are the main mechanisms that interweave to form the two-mode network. Through projection, the new one-mode network displays observed macro-structures: heterogeneity of strength and popularity among actors, high clustering, and community formation and segregation. 
of situations to happen - such as a family, a school, or a workplace - where relationships already exist ${ }^{2}$, For instance, in a workplace, relationships are created when someone new joins the firm. Then, social situations take place routinely, and colleagues interact by chatting during the coffee break or attending meetings, making these relationships evolve in a particular way. The existence of a relationship provides the means for interactions to happen in social situations that determine the quality and strength of the relationship, which in turn affects the quality of future situations and interactions, a dynamic process characterized by this feedback loop.

However, social situations can be endogenous or exogenous to circles..$^{3}$ Examples of endogenous situations are meetings in the workplace as mentioned above, group activities in a school class, or encounters among members of the same voluntary organization. Social circles prompt these situations to happen regularly with a relatively permanent set of actors, but not necessarily with all members of the circle in every situation, as in the example of group activity in a school class. Exogenous situations are those that happen independently of a circle's existence, such as a chat among acquaintances due to a casual encounter, or festivals such as rock concerts. These do not occur as frequently as endogenous situations. They also usually do not retain the same set of actors; but when they do, this set of actors can ultimately create new social circles.

Finally, we have social structure: the patterns of actors participating in social situations and circles, and the patterns of interactions (which happen in situations) and

\footnotetext{
${ }^{2}$ Note that I use the term relationship to represent the mere existence of a tie, with no implications regarding the intimacy or quality of the relationship. For instance, two teenagers in a school classroom have a relationship simply by attending the same class. In the context of a friendship network, their relationship might be irrelevant if they never talk to each other; but it might have relevance in the context of an infectious disease.

${ }^{3}$ I borrow the terms endogenous and exogenous from Block (8). However, here they have different meanings. First, I use them to define social situations instead of circles. Second, I use opposite definitions to those applied to endogenous and exogenous situations (or circles, in Block's case), due to our different approaches to network formation.
} 
relationships (which mostly arise from circles) between those actors. Situations and circles generalize the relations between individuals in the social structure, including dyadic relations: going out for a beer with a work colleague, and a couple living together, are examples respectively of a situation and a circle of size two.

\section{Modeling social structure with two-mode and projected networks}

Two-mode networks explicitly model individuals and their relations with collectivities; thus they are ideal for modeling actors' participation in social circles and situations. Mathematically, a two-mode network is a bipartite graph $B=\{U, V, E\}$, where $U$ and $V$ are disjoint sets of nodes, and $E=\{(u, v): u \in U, v \in V\}$ is the set of ties connecting the two types of nodes. Nodes of the set $U$ represent actors, and nodes of the set $V$ represent social circles and situations. No ties between nodes of the same set exist; when we want to study the relations between nodes of the same type, we create projections of the two-mode networks. A projection is a new one-mode network where nodes have ties between them if they have ties with a common node (of the other type) in the two-mode network (17). Here, as we are interested especially in the relations between actors, we focus on just one projection, where actors have ties with others if they participate in the same social circle. $4^{4}$ A one-mode network projected onto the set of actors $U$ is a graph $G=\{U, L\}$, where $L$ is the set of ties between actors. One-mode projected networks have different flavors, such as simple graph $G_{s}$, weighted graph $G_{w}$, and multigraph $G_{m}$ projections (19). I use concepts from these three types of projections to characterize social structure.

As we are dealing with two sets of nodes and two networks, the degree of a node has several distinct meanings:

\footnotetext{
${ }^{4}$ Projections onto the set of circles and situations are also possible. They can provide information, for instance, about how companies are related due to workers moving from one to the other, which is relevant for studies of knowledge transfers.
} 
- $d_{v}$ is the degree of node $v$ in $V$ in the two-mode network - the size of a social circle or situation.

- $k_{u}$ is the degree of node $u$ in $U$ in the two-mode network - the social activity of an actor 5

- $q_{u}^{s}=q_{u}^{w}$ is the degree of node $\mathrm{u}$ in the simple graph and weighted projected networks - the popularity of an actor.

- $q_{u}^{m}$ is the degree of node $\mathrm{u}$ in the multigraph projected network - the strength of an actor. Also, $q_{u}^{m}=\sum_{u^{\prime}} w_{u u^{\prime}}$, where $w_{u u^{\prime}}$ is the weight of the tie connecting actors $u$ and $u^{\prime}$ in a weighted projection.

When a pair of actors has ties with the same social circle for the first time (two-mode network), a tie between these actors comes into existence (one-mode projected network). The pair's subsequent joint participation in additional circles or situations forms cycles of size four - to which I refer as four-cycles - in the two-mode network, and represents the overlap mechanism. In the projection, either this pattern creates new ties (multigraph projection) or the tie weight increases by one (weighted projection). The number of ties and the tie weight between the pair of actors both represent the same thing: the strength of their relationship7 (Fig. S1).

\footnotetext{
${ }^{5}$ Social activity is the number of social circles and situations in which an actor participates. It is a result of an individual's agency, shaped by several variables, such as opportunity, resources, psychology, position in the social structure, and others.

${ }^{6}$ There exist several context-dependent ways of building weighted projected networks (see e.g. (20). When we say that the multigraph degree of an actor is equal to the sum of the weight of her ties, we are assuming a simple weighted projection, where each time a pair of actors participates in an additional social circle or situation, the tie weight increases by one.

${ }^{7}$ For simplicity, I refer to relationship strength as tie strength.
} 


\section{Degree heterogeneity does not emerge from preferential attach- ment.}

The proposed theory agrees with Block (8), who argues that preferential attachment, as a mechanism, is "at best secondary" due to its dependency on "data source, analysis method, and model parameterization." Moreover, psychological studies with children and adolescents in middle school suggest that students do not create bonds based on popularity $(21,22)$. Preferential attachment might play a leading role in systems where the node degree is a proxy for credibility or reliability (as in citation networks), which is not the case for popularity. In this theory of social structure, it functions in a different manner: instead of popular actors attracting others, some social circles do so, due to their size. For instance, a firm's size can represent stability, higher salaries, and more benefits; thus, it is relevant to an actor's choice to join that firm. However, even if it has a different nature, preferential attachment continues to be a secondary and contextdependent mechanism.

What breeds degree heterogeneity is the distribution of social activity modulated by the distribution of circle size and overlap. Let us develop this idea and say that Alice and Bob are siblings. To facilitate, let us consider that they go to the same school and attend the same classes, such that they have ties in common within their home (with their parents) and their classes (with their peers and teachers). Bob loves music, and apart from attending his classes, he plays in the school orchestra every day. Alice likes many things: after school, she practices indoor soccer on Mondays, goes to a book club on Tuesdays, and attends a Portuguese course on Wednesdays. If social activity were the only variable affecting popularity, Alice would surely have more relationships than Bob, as she is more socially active, participating in more circles. 
If we consider only the size of these extra social circles in which Alice and Bob participate (apart from their home and school classes), Bob has ties with, say, 15 peers in the orchestra, while Alice has ties with six others on the soccer team, four in the book club, and ten on her Portuguese course. With these numbers, Alice indeed has higher popularity. But if Alice would have decided to take private lessons instead of joining the Portuguese course, she would have lower popularity than Bob, even with higher social activity.

Now, let us assume that all of Alice's six peers on the soccer team are also on the Portuguese course with her, as the team will spend a training season in Brazil. The overlap between these two circles (several four-cycles in the two-mode network structure) results in Alice's popularity (the number of unique relationships equals 14) being lower than Bob's (15), despite her higher social activity and larger circles. Alice's strength is still higher (20 vs. 15), meaning that Alice has stronger ties with some of her alters than Bob has with his. Overlap creates differences between the strength $q_{u}^{m}$ (which includes repeated relationships) and the popularity $q_{u}^{s}$ of an actor (19), such that:

$$
q_{u}^{s} \leq q_{u}^{m}, \text { where } q_{u}^{m}=\sum_{j=1}^{k_{u}}\left(d_{v_{j}}-1\right) .
$$

That is, popularity is the sum of the number of actors $d_{v}$ in a social circle $v$ minus the actor herself, over all her $k_{u}$ social circles 8 reduced by the overlaps in which she is embedded; hence the inequality 9 In other words, popularity is the set of unique actors with which an individual has some sort of relationship, and social activity correlates positively with it $(23,24)$, modulated by circle size and overlap.

\footnotetext{
${ }^{8}$ This includes exogenous situations, which can also create relationships. However, as we will see later, relationships from exogenous situations are much rarer than from circles. Endogenous situations do not create relationships, as the circles in which they occur have already created those.

${ }^{9}$ Popularity and strength can only be equal with no overlap, which is unrealistic. Unfortunately, an analytical solution for this inequality considering overlap as a variable seems unfeasible.
} 
We can make similar analyses using previous empirical studies that have identified the heterogeneity of popularity but neglected social activity and circle size. Scientific collaborations, corporate boards, and movie productions are social systems that display right-skewed popularity and strength distributions (Fig. S2), and they function as convenient proxies for social structure, since they are also well modeled using two-mode and projected networks. When we bring together the distributions of popularity, strength, circle size, and social activity (Fig. S2), the correlation between social activity and popularity (Fig. S3), and the correlation between social activity and the average circle size of each actor (Fig. S4), we observe three main points. First, the difference between the popularity and strength distributions makes explicit the high levels of overlap. Second, social activity and average size do not show any correlation pattern, suggesting that most actors do not choose their social circles based on size; perhaps only a few with low social activity do so. Third, social activity and popularity correlate more in systems with lower heterogeneity of circle size. The chances of actors with low social activity ending up with high popularity are greater when the tail of the size distribution becomes heavier.

Circles size and actors' social activity are directly related. When an actor joins (or leaves) a circle, she increases (or decreases) both her social activity and that circle's size. Generalizing, we have $\sum_{v} d_{v}=\sum_{u} k_{u}$, that is, the sum of the social activity of all actors is equal to the sum of the size of all circles. This relation explicitly shows how social structure constrains social activity (and also popularity), especially due to institutionalized social circles with their limited sizes and demands - e.g. not every child can go to school, not every adult can have a job, and not every individual has the resources to participate in certain circles.

I propose that the composition of social circles is driven by dimensions of homophily. The standard approach to homophily involves only a pair of actors: "Homophily refers 
to the degree to which pairs of individuals who interact are similar with respect to certain attributes, such as beliefs, values, education, social status, etc." (25). If we broaden this approach and assign attributes to social circles, then we can think of the likelihood of actors participating in circles with similar attributes, as a result of the balance between agency and social structure: for instance, religious people attending religious services, a studious person going to a prestigious college, or slum-dwelling children joining drug-trafficking gangs, due to dimensions of homophily such as religion, education, and marginality respectively.

Actors participate in circles with attributes similar to their own, and it is likely that pairs of actors with similar attributes participate in more than one circle together, forming four-cycles; thus, overlap can be seen as a by-product of homophily. However, due to its relevance in shaping social structure, I prefer to treat overlap as a mechanism of network formation: its presence directly affects not only popularity (as we have seen), but also clustering and segregation.

\section{High clustering does not emerge from the triadic closure mecha- nism}

There are two significant problems when we consider that high clustering emerges from the triadic closure mechanism. The first relates to the formation of closed triplets; the second to the formation of open triplets.

For the first, we have a question that resembles the "chicken and egg" dilemma: we need to differentiate triadic closure between actors as a cause from triadic closure as

\footnotetext{
${ }^{10} \mathrm{I}$ use the term homophily in a broad sense, to include the position in the social structure and the restrictions it imposes on an actor's agency as a dimension of homophily. However, a discussion of the extent to which agency or social structure are determinant of actors' participation in circles and situations is beyond the scope of this study.
} 
an effect. The former is the widely known mechanism (also referred to as transitivity) that forms closed triplets "as a consequence of balance theory, whereby researchers have suggested that tendencies toward transitivity were the result of individuals seeking to make their ties cognitively consistent" (14). That is, if A and B are friends, and so are B and $\mathrm{C}$, then a friendship between $\mathrm{A}$ and $\mathrm{C}$ reduces the cognitive burden and avoids the pressure of imbalance in the personal networks of these three actors. Triadic closure as an effect results from the relationships within a social circle, where "clustering will most often be a result of the tendency of the foci to organize exclusive social interaction" (14). In this case, closed triplets appear solely due to several actors participating together in a social circle.

Let us work out an example again. Say that Alice has become an accountant in a small office but still plays soccer, so she belongs to two very distinct social circles (among others that she might participate in). The circle sizes are $d_{o}$ (office) and $d_{s}$ (soccer team), and their relationships create cliques also sized $d_{o}$ and $d_{s}$. Considering that the most common way of measuring transitivity is the global clustering coefficient given by

$$
C=\frac{\text { No. closed triplets }}{\text { No. closed triplets }+ \text { No. open triplets }}=\frac{\text { No. closed triplets }}{\text { No. total triplets }} \text {, }
$$

a clique is fully clustered. For instance, the soccer team has no open triplets and contributes $3\left(\begin{array}{c}d_{s} \\ 3\end{array}\right)=\frac{d_{s}\left(d_{s}-1\right)\left(d_{s}-2\right)}{2}$ closed triplets to the network. Following Feld's reasoning, all the closed triplets found in Alice's work and soccer circles result from the circles themselves - which foster repeated social situations - and not from the mechanism of triadic closure.

In turn, the circles' composition results from dimensions of homophily, as discussed above: one mostly due to educational background, and the other due to fondness for playing soccer. Alice is the bridge (or point of intersection) between these two distinct 
circles. Because of her, there are $\left(d_{o}-1\right)\left(d_{s}-1\right)$ open triplets. However, her colleagues at the office do not like the game, and her soccer peers have a different educational background, such that her alter pairs, made of one alter from each circle, are open triplets that are unlikely to close.

Now, the office is expanding and announces a new position that is filled by Clara, who is at first a stranger to everyone else in the workplace. As Clara starts her job, she creates relationships with all the office members - which will evolve according to future endogenous social situations - and closed triplets that result purely from homophily, without the triadic closure mechanism. Every time an actor is born and becomes part of a family, or an individual joins an new social circle with no previous ties to the circle's other members, $\frac{3\left(\left(d_{v}-1\right)\left(d_{v}-2\right)\right)}{2}$ closed triplets are created, without there even having been any open triplets in the first place. These are everyday processes and the sources of most relationships (15).

Like Alice, Clara enjoys playing soccer, and she accepts Alice's invitation to join the team. In this case, existing open triplets become closed. However, even when Clara joins a circle through Alice's influence, the cause of the closure of these triplets is not the triadic closure mechanism between individuals due to cognitive pressure. Instead, the cause is homophily once again: if Clara would have had different tastes in sport, these triplets would still be open. One's choice to become a member of a social circle results in triadic closure and popularity, driven by homophily, as I argued above 111 Supporting this reasoning, recently proposed network evolution models have achieved the generation of high clustering based on homophily and the neglect of the triadic closure mechanism $(3,10,26)$.

When Clara joins the soccer team, we have to face the second problem of dealing with high clustering due to the triadic closure mechanism. When interpreting Eq. (2),

\footnotetext{
${ }^{11}$ The illustrative example is clearly biased toward an actor's agency, but the reasoning also holds when participation in social circles is imposed or results from restricted agency.
} 
we tend to assume that the more closed triplets a network has, the higher its clustering coefficient is. However, to look further than the immediate structure, when Clara joins Alice's team, the clustering level might actually decrease, depending on the size of the two circles (Fig. 2)). When an individual becomes the second point of intersection of two cliques, new $\left(d_{v}-3\right)\left(d_{v^{\prime}}\right)$-with $d_{v} \geq d_{v^{\prime}}, d_{v} \geq 3$ - open triplets appear, a number that can grow faster than the $\frac{3\left(\left(d_{v}-1\right)\left(d_{v}-2\right)\right)}{2}$ closed triplets created.
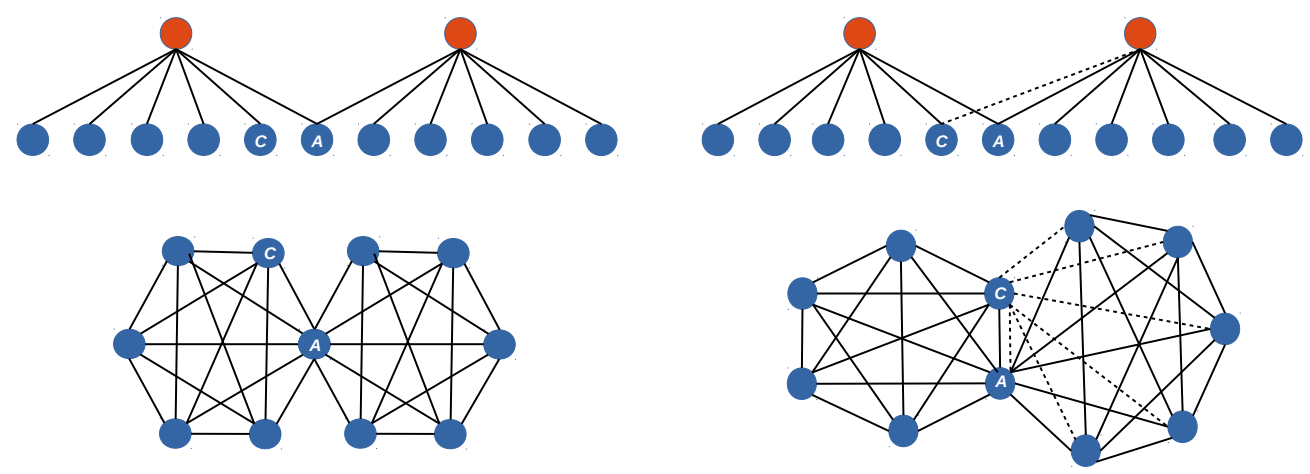

Figure 2: Diagrams of our toy social structure, with the two-mode network and its onemode projection, showing how the closure of triplets can decrease clustering. Before Clara joins the soccer team (social structure on the left), there are 120 closed and 25 open triplets, resulting in clustering $C=0.83$. After she joins the team (right), there are 165 closed and 40 open triplets, resulting in $C=0.80$. In this case, the slight shift to the right in the social activity distribution has resulted in lower clustering.

Similarly to heterogeneity of popularity, high clustering in social networks emerges from a balance between circle size and social activity distributions. These two distributions of the two-mode network have opposite effects on the structure of the one-mode projection: the more the size distribution shifts to the right, the more it favors closed triplets; the more the social activity distribution shifts to the right, the more it favors open triplets (27). Larger circles result in a more clustered social structure; several people being part of more circles results in a less clustered social structure. However, four- and 
six-cycles affect this balance.

Six-cycles are patterns that result in triadic closure of a different nature, where the three actors involved do not participate together in the same circle, thus deviating from balance theory. They result from dimensions of homophily and the composition of circles: if A and B have enough similar attributes to participate in the same circle, and the same is true for B and C's participation in another circle, then there might be a good chance that $\mathrm{A}$ and $\mathrm{C}$ will also have similar attributes to participate together in a third circle. These patterns are frequent (Table S1), can be seen as some sort of transitivity between dimensions of homophily, and might explain surprising properties in large-scale networks, such as the strong long-range ties found by Park, Blumenstock, and Macy (28).

While the effect of six-cycles is straightforward - they result in closed triplets in the one-mode projection, which increases clustering - four-cycles have a more concealed effect in regard to clustering (and also segregation, as we will see shortly), which we uncover through the analysis of synthetic networks (see supplementary materials, and Fig. 3b and 3e). Four-cycles help to keep clustering levels elevated by suppressing the formation of a higher proportion of open triplets (27). If Clara, instead of joining Alice's soccer team, would have opted for another circle, no overlap would exist, and the network clustering would be lower. A homogeneous society, in terms of dimensions of homophily, has high levels of overlap and is more clustered than a well-mixed society, even if they have comparable social activity and circle size distributions.

To momentarily shift our focus to short-term contexts, the triadic closure mechanism functions in exogenous social situations. Let us say that Alice decides to organize a party and invites people from the office and the soccer team. The party is a social situation in which all the open triplets between actors (that are present at the party) from the different circles finally close. These closures, however, last only as long as the social situation does. 
Once people leave the party, the ties cease to exist, and the triplets return to being open, as there are no social circles to nurture these relationships. Some of the triplets might close in the future, and the new ties might become long-term relationships. During the party, Daniel and Elle, from the office and the soccer team respectively, engage in conversation and decide to meet for lunch the next day. When they meet, the tie exists once more until that social situation is over - unless they meet regularly and, for instance, create their own social circle of size two by becoming a couple. If that happens, they have created a longterm relationship due to repeated situations and backed by a new social circle, such that their relationship might be meaningful in different contexts. Because relationships created in the same way as Daniel and Elle's are much less frequent than those created due to an
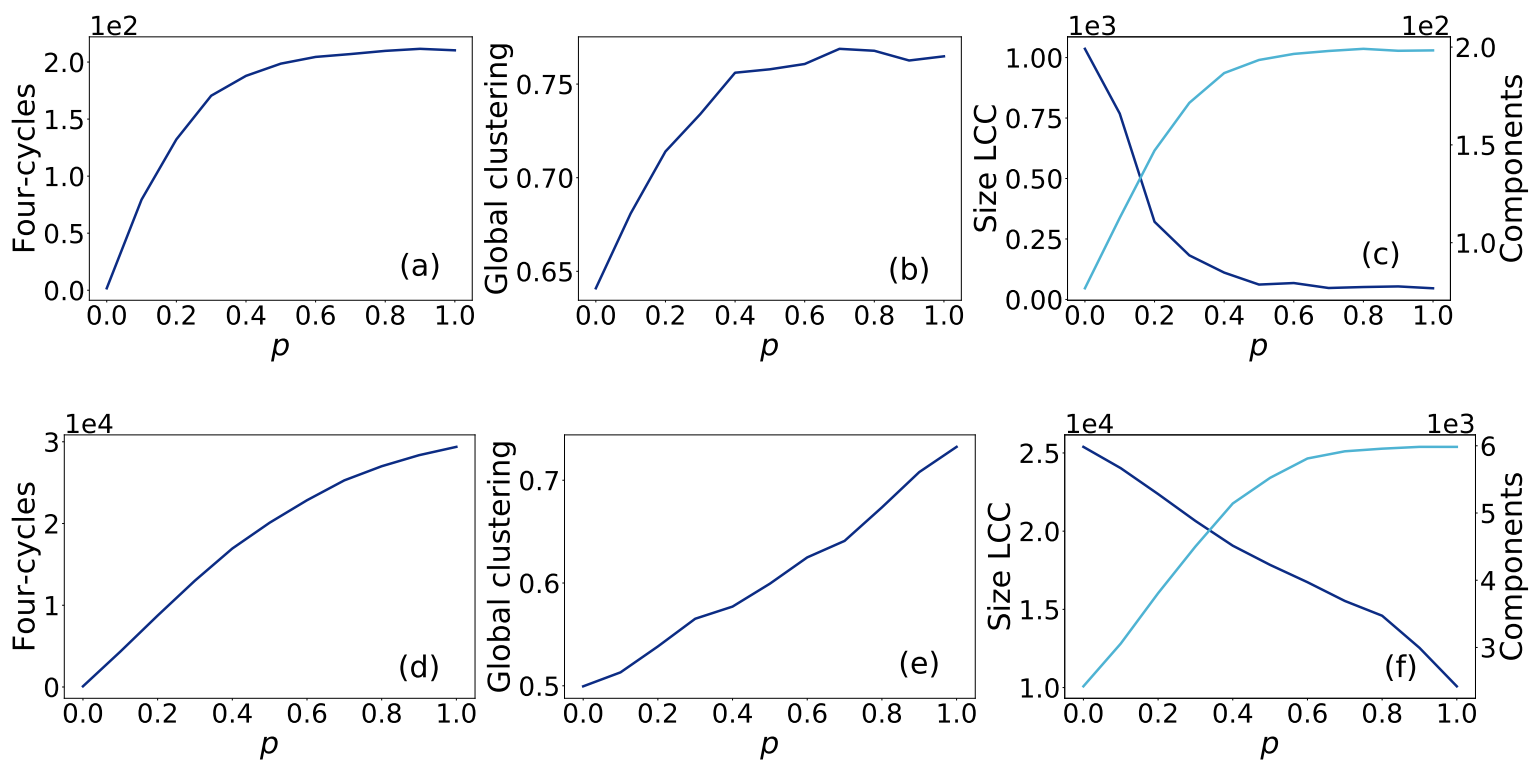

Figure 3: Effects of the $(\mathrm{a}) /(\mathrm{d})$ number of four-cycles on $(\mathrm{b}) /(\mathrm{d})$ clustering and $(\mathrm{c}) /(\mathrm{f})$ components of the network, using the Norwegian board of directors (top) and arXiv biology (bottom) networks as empirical references for the configuration model (see supplementary materials and methods). As the number of four-cycles increases following a probably p of overlap, so does (b)/(d) clustering and the (c)/(f) number of components (light blue) in the network. In contrast, the (c)/(f) largest component (dark blue) of the network becomes smaller. Segregation and clustering increase with more overlap. 
existing social circle, triadic closure is another secondary mechanism of social structure. It does not have the capacity by itself to generate highly clustered networks.

\section{Overlap increases segregation}

Homophily is one of the leading mechanisms of network formation, and it is seen as the fundamental cause of social segregation. But how does segregation emerge from a network standpoint?

In order to address segregation as a "form of isolation which places limits upon contact, communication, and social relations" $(29,30)$, I restrict myself to looking at network components - sets of actors that are disconnected from other sets in the network - that explicitly represent the given definition of segregation.

Some dimensions of homophily induce actors to be part of several circles, and some reinforce others, such as ethnicity and religion. When circles become more homogeneous thanks to a few dimensions, pairs of actors have joint participation in several social circles, creating stronger ties between them. Time, energy, and cognition limit one's ability to be part of as many circles as one wants. As a consequence, stronger ties generate not only clustering but also segregation. The rationale is simple: if an actor overlaps too much with a set of alters, she does not have the resources to create relationships with others.

To test this hypothesis, I use synthetic networks built with different levels of overlap, but I retain the same social activity and circle sizes as in empirical networks (see supplementary materials). As the presence of four-circles increases, so does the number of disconnected components in the network. Moreover, the size of the largest component

drastically decreases with overlap, showing that the network becomes more segregated (Fig. 3c and 3f). Overlapping amplifies the strength of ties, clustering, segregation, and the difference between the strength and popularity of actors (Fig. 4). 


\section{Social circles vs. social situations and the quality of relationships}

Although we can represent circles and situations as the same type of node in the two-mode network, there exist fundamental differences among them: circles create relationships; endogenous situations affect how these relationships evolve; exogenous situations do both, but at a much lower frequency. Endogenous situations are particularly important for the relationship dynamics within a circle, depending on the size of that circle.

At the same time that right-skewed circle size distributions limit the role of social activity in popularity, they can present themselves as limitations for the modeling of social structure with two-mode networks, depending on the data collection and study design. If we take the empirical data we have used here as proxies for social structure, the problem lies in considering that every scientific collaboration, movie production, or corporate board has the same effect on the tie quality between actors, regardless of size. Feld (14) too noted this: social circles have different constraints-i.e. how a social circle enforces the evolution of relationships between its members - that decay with size. The larger the circle, the less meaningful a relationship between its members might be, and

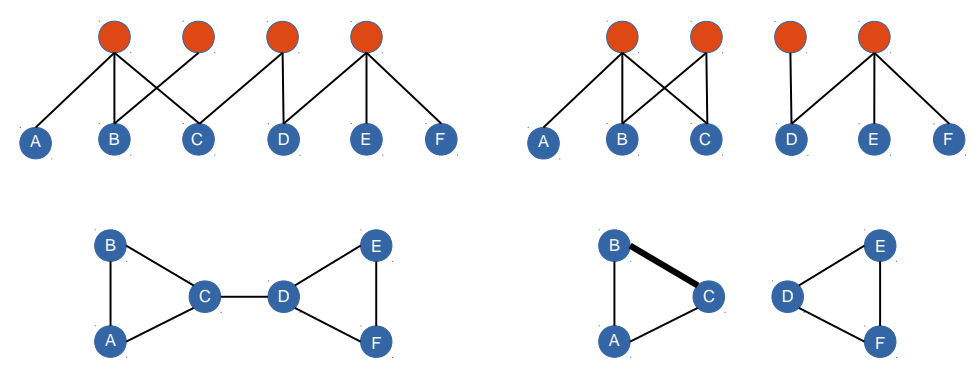

Figure 4: Diagram of how overlap increases the strength of a tie (between nodes B and C), clustering, segregation, and the difference between the strength and popularity of actors, retaining the same circle size and social activity distributions. 
the more likely it is that endogenous social situations will involve small subsets of actors in that circle, also creating heterogeneity in tie quality and strength.

Leaving aside the size constraint of circles leads to an "inflation" (31) of ties in the projected network. A social circle of size $d_{v}$ induces a clique of relationships of the same size with $\frac{d_{v}\left(d_{v}-1\right)}{2}$ ties. It is reasonable to assert that, in a family of four, each member has a meaningful relationship with every other (six ties) in several contexts; for a school with 2,000 students (around 2 million ties), it is impossible to think that all these ties will have useful meaning across different studies. Thus, inflation is a subjective matter and a problem of study design and data collection, not projections. In studies of friendship networks (for which Feld proposed his focus theory), where intimate relationships are more relevant, social circles must have higher constraints. That means choosing a class instead of the whole school, or a department instead of the whole company, where the size of endogenous situations are closer to that of the circle. ${ }^{12}$ For studies where even isolated exogenous social situations are relevant-for example, studies that model the spread of infectious diseases - social circles can be larger with lower constraints, and hierarchical circles can be considered. Understanding the dynamics of endogenous situations is also crucial for the latter case, where circles are embedded in others.

Embedded social circles also happen when they are relatively small. For instance, two persons that become a couple create their own circle of size two. When they decide to introduce each other to their respective families, we can think of these events as each individual joining the family social circle of the other. In the two-mode network representation, the couple is attached to three circles together, creating four-cycles (three four-cycles, in this case) of which the couple is part. In the projection, this automatically results in a stronger tie between the two, even in a scenario that dismisses situations.

\footnotetext{
${ }^{12}$ Another approach to dealing with tie inflation in some contexts is to find statistically significant ties in weighted projections $(32,33)$.
} 
When situations are included, this arrangement represents more opportunities for interaction between the two actors that form the couple: they have more chances of participating in social situations together - strengthening even more their tie - than one of them has with another member of their families.

A circle's characteristic is yet another relevant variable for constraints, determining how endogenous situations happen and the quality of ties. Institutionalized circles (educational, religious, governmental, and so on) are deeply rooted in the social structure, are practically permanent-lasting for years, or even centuries - and are part of everyone's daily life. The endogenous situations within each of these institutional circles have different dynamics, due to the circle's constraint, that will result in distinctive tie qualities between pairs of actors. For example, a pair working in a court of justice is expected to have a different tie quality from a pair attending religious services together, even if the frequency of situations (and therefore the tie strength) in which these two pairs of actors participate in their respective social circles is the same.

Finally, the endogenous situations of the same circle can also differ widely. A political party is a good example where the actors participate together in a circle of common interests and goals, but where internal disputes and antagonistic views generate a mix of positive and negative situations. The actors in the party might have a homogeneous tie strength distribution among them, but almost certainly the tie quality distribution will be heterogeneous.

\section{Implications}

I have proposed a theory in which relationships - and their quality - between individuals stem from their participation in social circles and how they interact in endogenous (withincircles) or exogenous (outside-circles) social situations. We can explicitly model this 
theory using two-mode networks and projections. Two-mode networks generalize social relationships in circles and situations of any size, including of size two, representing dyadic relations.

The theory explains how macro-level properties of social structure - the heterogeneity of strength and popularity among actors, high clustering, and community formation and segregation - emerge predominantly from homophily, social activity, and overlap working in conjunction. It also downgrades preferential attachment and triadic closure, long regarded as primary mechanisms of social network formation.

The understanding that social circles and situations are determinant of an actor's set of ties and the quality of her relationships has direct implications for several social processes, and for policy-making.

When it comes to social inclusion and the democratization of economic and social capital, we can pose new questions to try to answer old ones. We have seen that participation in circles is crucial for segregation. How can we adapt existing circles so as to produce less segregated societies? To what extent can circles avoid obstacles to the navigation of our social networks' core-periphery structure? How can one have more access to opportunities by building long-range ties based on such circles? These are all questions that offer the possibility of modeling agency with restrictions in the social structure due to its institutionalized social circles.

Regarding social tolerance, Mousa (34) has recently found that two sets of people that are distinct due to their religious beliefs can build tolerance and cohesion if they mix in a social circle with a common dimension of homophily (soccer), albeit with limited effects. Her findings support this theory's predictability. Religion is a dimension that induces membership of several circles, resulting in overlap and strong ties between actors. A social circle of soccer players creates relationships, but it cannot by itself build ties 
strong enough to overcome the effects of many other circles. Thus, religion will continue to build close-knit communities and segregation, unless more circles with other dimensions of homophily attract the actors' limited social activity. Actors would have to leave behind homogeneous circles and join heterogeneous ones to balance the strength of their ties with actors of different religions.

In the realm of infectious diseases, studies exploring transmission through encounter networks (35) can be improved by acknowledging that the frequency of contacts is a result of the actor's social activity and circles. Refined models of social structure considering social circles can aid us to find more precise disease-induced herd immunity levels (36), facilitating the implementation of social-distancing and contact-tracing measures (37), and providing alternatives to business and school closures (38). In general, more social activity leads to a higher frequency of weak ties, facilitating the emergence of epidemics, while large circles with overlap lead to a higher frequency of strong ties, favoring endemics.

These are just a few examples of the multitude of social processes that could beneficially be studied through the angle proposed by this theory.

\section{Acknowledgments}

The author would like to thank Patrick S. Park for comments and advice. 


\section{References}

[1] M. T. Rivera, S. B. Soderstrom, B. Uzzi, Annu. Rev. Sociol. 36, 91 (2010).

[2] G. Gilbert, L. Hamill, JASSS 12 (2009).

[3] F. Papadopoulos, M. Kitsak, M. Á. Serrano, M. Boguná, D. Krioukov, Nature 489, 537 (2012).

[4] J. Skvoretz, Am. J. Sociol. 119, 486 (2013).

[5] C. Stadtfeld, Emerging Trends in the Social and Behavioral Sciences: An Interdisciplinary, Searchable, and Linkable Resource pp. 1-15 (2015).

[6] T. A. Snijders, C. E. Steglich, Sociol. Method. \& Res. 44, 222 (2015).

[7] Q.-M. Zhang, X.-K. Xu, Y.-X. Zhu, T. Zhou, Sci. Rep. 5, 10350 (2015).

[8] P. Block, Sociol. Sci. 5, 402 (2018).

[9] A. Asikainen, G. Iñiguez, J. Ureña-Carrión, K. Kaski, M. Kivelä, Sci. Adv. 6, eaax7310 (2020).

[10] S. Talaga, A. Nowak, JASSS 23 (2020).

[11] A. Wimmer, K. Lewis, Am. J. Sociol. 116, 583 (2010).

[12] C. Kadushin, Am. Sociol. Rev. pp. 786-802 (1966).

[13] E. O. Laumann, Bonds of pluralism: The form and substance of urban social networks (New York: J. Wiley, 1973).

[14] S. L. Feld, Am. J. Sociol. 86, 1015 (1981).

[15] M. McPherson, L. Smith-Lovin, J. M. Cook, Annu. Rev. Sociol. 27, 415 (2001).

[16] M. Grossetti, Soc. Networks 27, 289 (2005).

[17] R. L. Breiger, Soc. Forces 53, 181 (1974).

[18] R. A. Hinde, Man pp. 1-17 (1976).

[19] D. Vasques Filho, D. R. O’Neale, Phys. Rev. E 98, 022307 (2018).

[20] M. Coscia, L. Rossi, 2019 IEEE/ACM International Conference on Advances in Social Networks Analysis and Mining (ASONAM) (IEEE, 2019), pp. 286-293.

[21] J. T. Parkhurst, A. Hopmeyer, J. Early Adolesc. 18, 125 (1998).

[22] K. M. Lafontana, A. H. Cillessen, J. Genet. Psychol. 160, 225 (1999). 
[23] M. Krantz, Child Dev. pp. 376-379 (1982).

[24] P. J. LaFreniere, L. A. Sroufe, Dev. Psychol. 21, 56 (1985).

[25] E. M. Rogers, D. K. Bhowmik, Public Opin. Quart. 34, 523 (1970).

[26] D. Vasques Filho, D. R. O'Neale, International Conference on Network Science (Springer, 2020), pp. 3-16.

[27] D. Vasques Filho, D. R. O’Neale, Phys. Rev. E 101, 052305 (2020).

[28] P. S. Park, J. E. Blumenstock, M. W. Macy, Science 362, 1410 (2018).

[29] B. Berry, Race and ethnic relations. (Boston: Harghton Mifflin Company, 1958).

[30] L. C. Freeman, Socio. Method. Res. 6, 411 (1978).

[31] M. Latapy, C. Magnien, N. Del Vecchio, Soc. Networks 30, 31 (2008).

[32] Z. Neal, Soc. Networks 39, 84 (2014).

[33] J. Liebig, A. Rao, Europhys. Lett. 113, 28003 (2016).

[34] S. Mousa, Science 369, 866 (2020).

[35] L. Danon, T. A. House, J. M. Read, M. J. Keeling, J. R. Soc. Interface 9, 2826 (2012).

[36] T. Britton, F. Ball, P. Trapman, Science 369, 846 (2020).

[37] S. M. Kissler, C. Tedijanto, E. Goldstein, Y. H. Grad, M. Lipsitch, Science 368, 860 (2020).

[38] G. St-Onge, V. Thibeault, A. Allard, L. J. Dubé, L. Hébert-Dufresne, arXiv preprint arXiv:2003.05924 (2020).

[39] Vasques Filho, D. and O’Neale, D. R. (2020). J. Complex Netw., 8(4):cnaa016.

[40] Seierstad, C. and Opsahl, T. (2011). Scand. J. Manag., 27(1):44-54.

[41] Evtushenko, A. and Gastner, M. T. (2019). In International Conference on Complex Networks and Their Applications, pages 586-598. Springer.

[42] Auer, S., Bizer, C., Kobilarov, G., Lehmann, J., Cyganiak, R., and Ives, Z. (2007). In The Semantic Web, pages 722-735. Springer.

[43] Barabási, A.-L. and Albert, R. (1999). Science, 286(5439):509-512. 
Supplementary Materials for

Mechanisms and emergent properties of social structure: the duality of actors and social circles

Demival Vasques Filho

Email: vasquesfilho@ieg-mainz.de

Materials and Methods

Figs. S1 to S4

Table S1

References (39-43) 
Materials and methods - Contents

\begin{tabular}{lll}
\hline 1 & Mechanisms of network formation & S3
\end{tabular}

2 Synthetic networks with tunable overlapping $\quad$ S5

$\begin{array}{lll}3 & \text { Data } & \text { S6 }\end{array}$

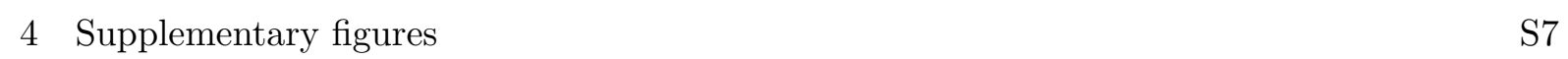




\section{Mechanisms of network formation}

The multidisciplinary nature of research on social systems has created a word salad of terminology and jargon, all broadly defined. Below, I list the main concepts that appear in this article and describe their meaning for the proposed discussion.

In their comprehensive review, Rivera, Soderstrom, and Uzzi [1] classify the mechanisms of network evolution into three categories - assortative, relational, and proximity — each with subdivisions named dynamics. What they call dynamics, I simply call mechanisms, thereby avoiding their hierarchical classification, which seems unnecessary and gives the impression that mechanisms of network formation function independently. Nevertheless, I use their work as the basis for my own definitions of the mechanisms that are relevant to this work, drawing parallels between the terms and meanings found in the literature and how I propose to use them here. I will restrict myself to defining the concepts objectively and briefly, without reviewing their historical usage. First I will define some terms used throughout this article, then I will put them in the context of the mechanisms.

\section{Sociometric terms}

- Popularity - the number of different alters an actor has, in terms of interactions (for social situations) or relationships (for social circles). It may be a sum of both, depending on the context.

- Overlap - the joint membership of a pair of actors in two social circles or situations.

- Actor strength - the sum of the strength of all ties connected to an actor. The strength of a tie is given by the level of overlap between a pair of actors.

- Degree (popularity) heterogeneity - high variance in the popularity of actors, usually due to a right-skewed (heavy-tailed) distribution: low popularity for most actors, high popularity for a few.

- Triplet (triad) - a set of three nodes, which can be connected by either two ties (open triplets) or three ties (closed triplets).

- Clustering - the proportion of closed triplets to the total number of triplets in the network.

- Community - a macro-scale structure consisting of a set of actors that are more densely connected among themselves than they are to actors from another set.

- Clique - a fully connected subgraph, that is, a set of actors that have ties to every other actor in the set.

Mechanisms

- Preferential attachment - the mechanism by which one that has much gains more. Regarding relationships, it means that actors attract ties according to their popularity: popular (high-degree) actors attract more ties than unpopular (low-degree) ones. I also use this term to address cases where a large social circle can attract more people due to its size. Other names for this mechanism are "the Matthew effect," cumulative advantage, the Yule 
process, the popularity mechanism, dynamics of degree, preferential linking, and probably others.

- Triadic closure - the mechanism of forming closed triplets, that is, if A is connected to B and $\mathrm{C}$, then $\mathrm{B}$ and $\mathrm{C}$ tend to have a tie between themselves at some point. Another term used for this mechanism is transitivity.

- Homophily - pairs of actors with similar attributes (dimensions of homophily) are more likely to have a tie between them. The proposed theory expands the concept of homophily to social circles and situations, such that an actor with similar attributes to those of a social circle is more likely to participate in it. Homophily is also known as assortativity.

- Social activity - the number of social circles (or situations) in which an actor participates. The theory introduces social activity as a mechanism of network formation, arguing that homophily, social activity, and overlap are the leading mechanisms of social structure. Degree heterogeneity, high clustering, and community formation emerge, directly or indirectly, from these two mechanisms.

- Repetition - although the scholarship considers repetition to be a mechanism [1], the present theory suggests that it is the result of overlap and homophily. That is, a pair of actors that have many dimensions of homophily in common will likely be part, together, of many social circles and situations, increasing the strength of the tie between this pair.

- Reciprocity - this mechanism implies a directed network. I argue that it is a secondary mechanism, because it depends on the context. It might be relevant, for instance, for friendship networks. But social structure is more than that and, as a more general and abstract perspective, does not include directional ties. Moreover, we cannot associate any macro-level structure with reciprocity. 


\section{Synthetic networks with tunable overlapping}

To uncover the effects of overlap (four-cycles) on the structure of the projected network, I propose a version of the configuration model for two-mode networks, but with tunable levels of overlap. The configuration model retains the degree sequence of both sets of nodes found in empirical two-mode social networks - i.e. it retains the size of social circles and the social activity of actors - and randomly rewires the ties between actors and circles [39].

This rearrangement of a traditional configuration model works as follows: first, we assign to each circle or actor the number of available ties it has, according to its degree in the empirical two-mode network. Second, we choose a circle at random and subtract one from the number of available ties. Third, we choose an actor at random and also subtract one from the available ties. Finally we connect the actor to the social circle. We repeat this step, always picking actors and circles at random (providing they still have available ties left) and connecting them. Random arrangements like this produce little overlap when compared with the original empirical network [39].

To overcome this, we introduce a probability $p$ with which the randomly chosen circle tries to create a four-cycle: that is, with probability $(1-p)$, we execute the same step as described before. When the circle tries to create a four-cycle, it looks to its members (first neighbors in the two-mode network), then to other circles in which these members participate (second neighbors), then to the members of these other circles (third neighbors), and then it tries to force one of these last members to become its own member, closing the four-cycle.

As we increase $p$ and the number of four-cycles in the bipartite network, we reveal two important effects on the structure of the projected network: higher clustering, and a higher number of connected components (Fig. 4). 


\section{Data}

The scale and richness of data necessary to recreate social structure makes the performance of empirical studies very challenging, such that we have to rely on data sets that represent only small parts of the whole social network and entail several limitations. Social data collected in a way that allows the use of two-mode networks are rare, due to the focus of social network research on one-mode networks. Also, many available data sets are either too small or too large, making it difficult to perform some of the analysis here.

I use three types of data, with two data sets of each type. The first type represents scientific collaborations on papers in biology and mathematics [27]. I build two-mode networks connecting authors to papers they have published; the one-mode projections are the coauthorship networks, where two authors are connected if they appear in the same paper. The second type represents individuals that occupy positions on the boards of directors of firms in Norway [40] and globally [41]. Nodes in the two-mode network are individuals and corporate boards, with a tie between them if one sits on the board. The projections on the directors' set of nodes create networks of codirectors. The third type represents actors that are members of movie casts, from the DBpedia [42] and IMDb [43] databases. The one-mode projections are the costarring networks. Table S1 presents a summary of the features of the two-mode and projected networks built from these data.

Table S1: Network descriptives for the arXiv biology, arXiv mathematics, Norwegian board, international board, DBpedia film actor, and IMDb film actor networks. The table presents the numbers of authors, circles, and ties in the two-mode networks, four-cycles, and six-cycles; mean actors' popularity and strength; global clustering; size of the largest connected component (LCC); and number of components of the projection.

\begin{tabular}{|l|ll|ll|ll|}
\hline Properties & Bio & Maths & Nw. & Intl. & DBpedia & IMDb \\
\hline No. actors & 29,734 & 152,792 & 1,422 & 321,869 & 76,098 & 127,823 \\
No. circles & 17,281 & 300,686 & 355 & 34,769 & 81,085 & 383,640 \\
No. ties & 54,001 & 599,387 & 1,748 & 376,918 & 281,396 & $1.5 \times 10^{6}$ \\
No. four-cycles & 34,659 & 515,144 & 92 & 79,885 & 221,363 & $3.5 \times 10^{6}$ \\
No. six-cycles & 5,272 & 263,487 & 7 & 12,286 & $3.9 \times 10^{6}$ & $1.0 \times 10^{9}$ \\
Mean popularity & 6.3 & 3.99 & 5.43 & 16.09 & 64.17 & 320.6 \\
Mean strength & 7.4 & 6.19 & 5.56 & 16.36 & 68.35 & 358.7 \\
Clustering & 0.84 & 0.35 & 0.68 & 0.78 & 0.43 & 0.22 \\
Size of LCC & 14,113 & 109,001 & 848 & 240,794 & 67,652 & 124,414 \\
No. components & 3,757 & 23,181 & 126 & 9,367 & 7,055 & 3,142 \\
\hline
\end{tabular}




\section{Supplementary figures}

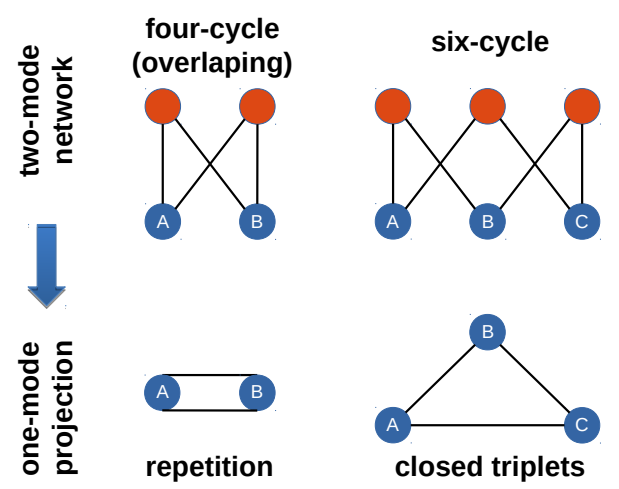

Fig. S1: Diagram showing how four- and six-cycles in the two-mode network result in stronger ties and closed triplets respectively in the one-mode projected network. When one chooses a simple graph projection, the two links between A and B are amalgamated into one; when the projection is weighted, the tie will have a weight that can be proportional to the number of four-cycles to which A and B jointly belong. 

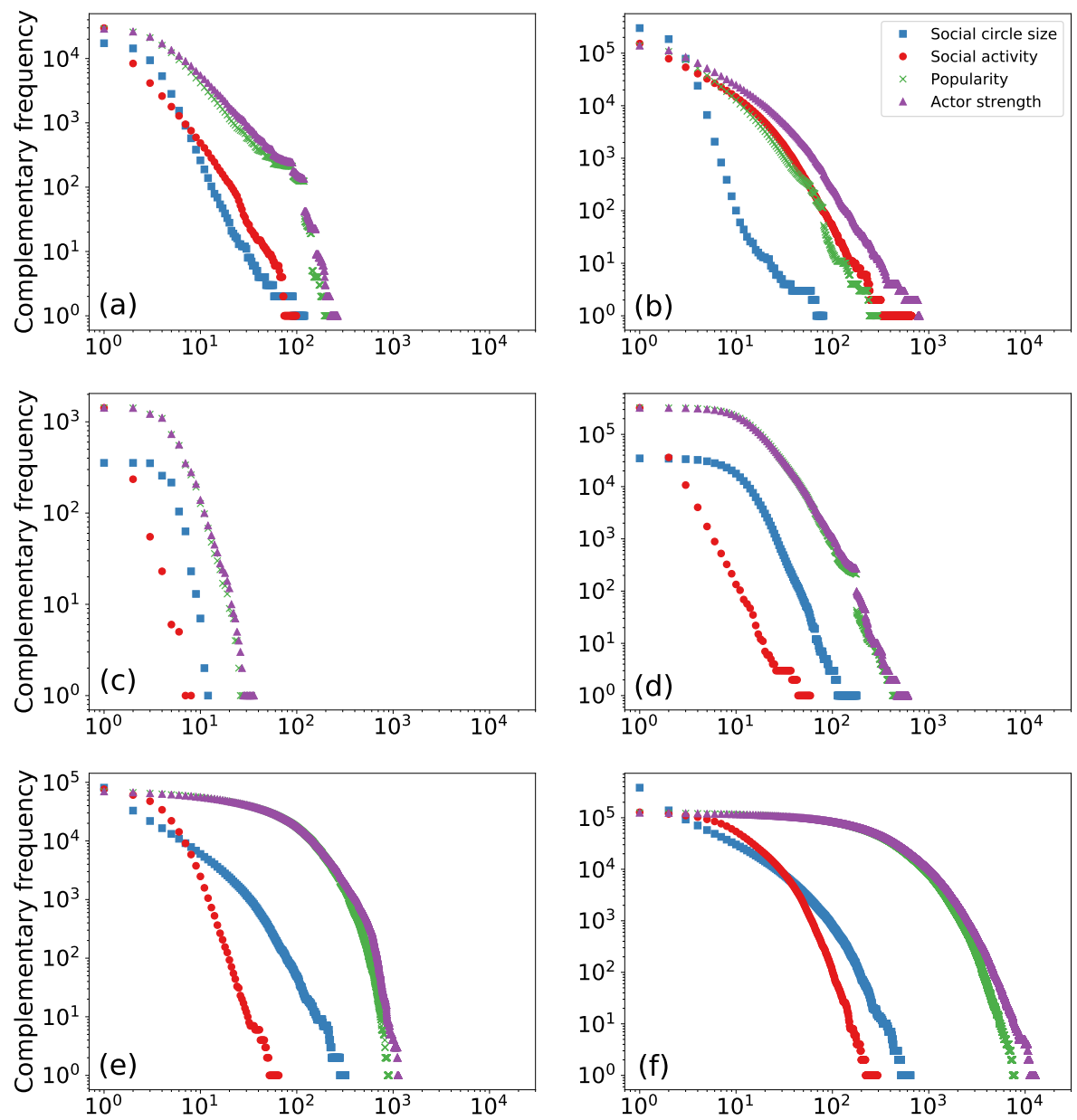

Fig. S2: Degree distributions for (a) biology, (b) math, (c) Norwegian boards, (d) global boards, (e) movies in DBpedia, and (f) movies in IMDb. The degrees represent: size of social circles (blue dots) and number of memberships (red squares) in the two-mode networks, and degree (green cross) and strength (purple triangle) of individuals in the weighted and multigraph onemode projections respectively. 

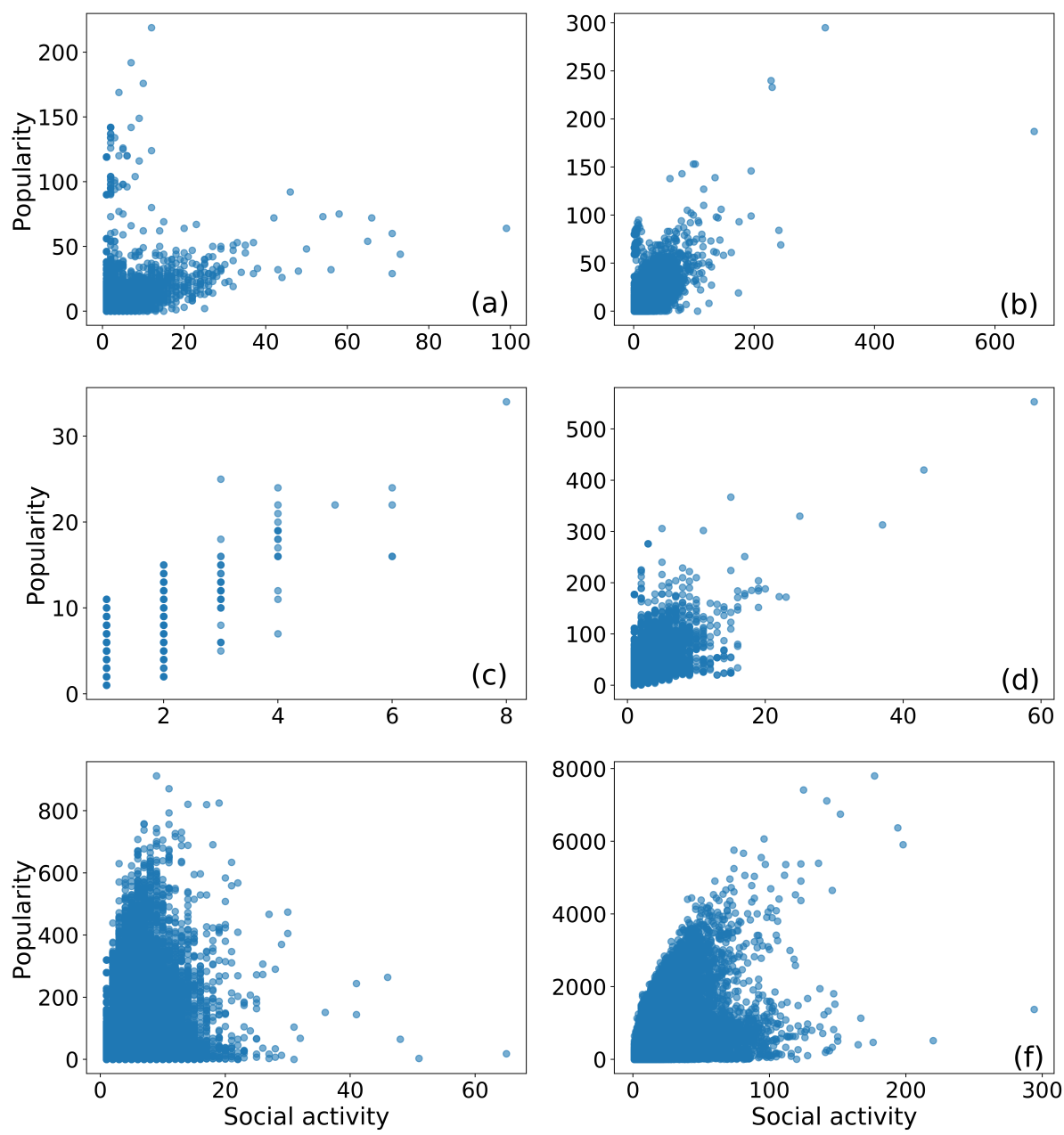

Fig. S3: Correlations between the degree of the individuals and their number of memberships in social circles for (a) biology, (b) math, (c) Norwegian boards, (d) global boards, (e) movies in DBpedia, and (f) movies production in IMDb. All cases have positive correlations, but the correlation is stronger for those with less heterogeneity in the size of social circles (Fig S2). As the tail of the distribution of social circles size gets heavier, the probability of an individual joining a large social circle becomes higher, which results in individuals with low social activity but high degree in the one-mode projection. 

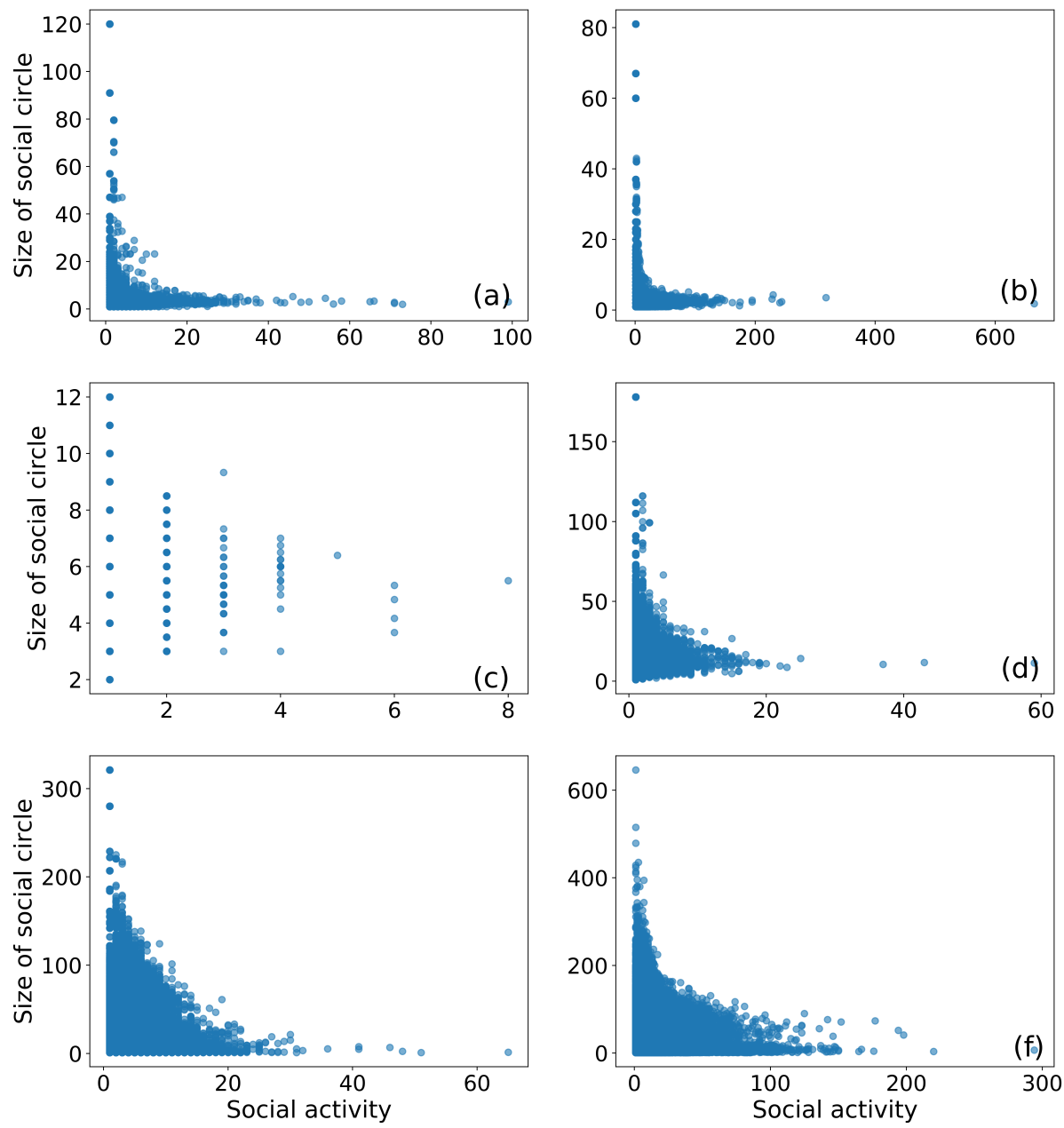

Fig. S4: Correlations between the degree of the individuals and the average size of their social circles for (a) biology, (b) math, (c) Norwegian boards, (d) global boards, (e) movies in DBpedia, and (f) movies in IMDb. All cases show these two variables are uncorrelated, suggesting that individuals join social circles without a well-defined strategy of searching for large ones. 